\section{hommes}

\section{Hommes \& migrations}

Revue française de référence sur les dynamiques

migratoires

$1312 \mid 2015$

Diasporas iraniennes

\title{
Élections à distance et politique diasporique
}

Le cas de la diaspora iranienne au Canada

\section{Leila Koochakzadeh}

\section{(2) OpenEdition}

12 Journals

\section{Édition électronique}

URL : http://journals.openedition.org/hommesmigrations/3490

DOI : 10.4000/hommesmigrations.3490

ISSN : 2262-3353

Éditeur

Musée national de l'histoire de l'immigration

\section{Édition imprimée}

Date de publication : 1 octobre 2015

Pagination : $43-49$

ISBN : 978-2-919040-33-9

ISSN : 1142-852X

Référence électronique

Leila Koochakzadeh, «Élections à distance et politique diasporique», Hommes \& migrations [En ligne], 1312 | 2015, mis en ligne le 01 octobre 2018, consulté le 02 mai 2019. URL : http://

journals.openedition.org/hommesmigrations/3490; DOI : 10.4000/hommesmigrations.3490 


\section{ÉLECTIONS À DISTANCE ET POLITIQUE DIASPORIQUE LE CAS DE LA DIASPORA IRANIENNE AU CANADA}

par LEILA KOOCHAKZADEH, doctorante en histoire, université de Montréal¹.

La suspension des relations diplomatiques entre

le Canada et I'Iran en 2012 a eu de graves conséquences sur

la capacité d'action politique de la diaspora iranienne résidant au Canada. Empêchés de prendre part à l'élection présidentielle de 2013 en Iran, marquée par la victoire d'Hassan

Rohani, les Iraniens du Canada se sont mobilisés pour faire entendre leurs voix. Dans la communauté iranienne de Toronto, par exemple, sont apparus de profonds clivages idéologiques entre les défenseurs du droit de vote à distance et les opposants plus radicaux au régime en place.

Si les phénomènes migratoires concernant les Iraniens sont anciens, l'émigration massive vers le Canada date de la révolution islamique de 1979. D’après une enquête nationale de 2011, le nombre d'Iraniens présents au Canada est estimé à $163290^{2}$, ce qui en fait, à ce jour, l'une des principales communautés iraniennes hors d'Iran.

Les relations diplomatiques entre l'Iran et le Canada ont débuté en 1955. D’abord globalement fluides, elles se limitaient aux échanges commerciaux et culturels. Après 1979, elles sont devenues plus chaotiques. Néanmoins, jamais la tension entre les deux pays n'a été si forte que lors de la rupture diplomatique de septembre 2012. Le
7 septembre 2012, le gouvernement canadien a, en effet, suspendu ses relations avec l'Iran, décrétant que le gouvernement iranien constituait "la menace la plus importante à la paix et à la sécurité mondiale $3^{3}$. John Baird, ministre canadien des Affaires étrangères, a ainsi ordonné la fermeture immédiate de l'ambassade du Canada à Téhéran. Les diplomates iraniens en poste à Ottawa, déclarés persona non grata ${ }^{4}$, ont reçu l'ordre de quitter le territoire dans un délai de cinq jours. Cette décision radicale n'était pourtant pas allée sans critiques de la part même des anciens ambassadeurs canadiens en Iran ${ }^{5}$. Il ne serait peut-être pas exagéré de dire que la diaspora iranienne est la plus concernée par 
cette rupture diplomatique, compte tenu de ses effets directs sur la vie quotidienne des Iraniens au Canada ${ }^{6}$.

\section{La diplomatie directe du Canada en question}

Pour compenser son absence diplomatique en Iran, le gouvernement canadien a tenté de mettre en place une autre forme de rapprochement avec les Iraniens eux-mêmes, une "diplomatie directe", selon l'expression canadienne, et ce, huit mois après la rupture des relations diplomatiques entre les deux pays. Le ministère des Affaires étrangères canadien a créé un site inter-

Bien que l'Iran compte parmi

les pays qui attribuent

le droit de vote à distance

à leur diaspora, il n'a pourtant

toujours pas mis en place

d'initiatives telles que le vote électronique ou le vote postal. net en anglais et en persan permettant aux internautes iraniens de communiquer directement avec le gouvernement canadien "sur divers sujets qui les touchent", pour un coût de 3,2 millions de dollars canadiens ${ }^{8}$. Afin de mener à bien ce projet intitulé "le dialogue mondial sur l'avenir de l'Iran", la Munk School of Global Affairs de l'université de Toronto a collaboré avec le gouvernement canadien ; elle a organisé des réunions à travers lesquelles des membres de la diaspora iranienne discutent de sujets tels que l'innovation technologique, l'esprit d'entreprise, la société civile, etc. La réunion d'ouverture a eu lieu le 10 mai 2013, avec le discours de John Baird, alors ministre des Affaires étrangères du Canada. Conscient d'une certaine contradiction entre une telle initiative et la rupture diplomatique opérée par le Canada, John Baird a tenté de se justifier :
"Je sais que ce message peut sembler paradoxal de la part d'un gouvernement qui a rompu ses relations avec le régime l'an dernier. Toutefois, notre décision de fermer l'ambassade du Canada à Téhéran ne visait pas le peuple iranien (...). Il s'agissait d'assurer la protection de nos représentants courageux, femmes et hommes, qui servaient leur pays dans la paix."

Son discours d'ouverture d'une "diplomatie directe" et la réunion qui s'en est suivie ont abordé la question de l'élection présidentielle en Iran. John Baird a insisté notamment sur le fait que les élections en Iran ne sont pas "libres et justes" et il a demandé à la communauté internationale de suivre l'exemple canadien, afin d'accentuer la pression sur le régime iranien. Outre l'initiative en elle-même, la date et les modalités d'organisation de cette "diplomatie directe" ne sont pas sans ambiguïtés : sa mise en place a eu lieu en plein scrutin présidentiel en Iran, alors que, durant les huit mois écoulés depuis la rupture, les manifestations d'une volonté de normalisation des relations de la part d'Iraniens du Canada ou d'Iran s'étaient multipliées. Les buts politiques de cette "diplomatie directe", loin de rendre service au peuple iranien, sont en fait évidents et se situent dans la droite ligne de la rupture avec le régime iranien.

\section{Le droit de vote à distance et l'élection présidentielle iranienne de 2013}

L'attribution du droit de vote à distance pour la diaspora remonte à 1902, année où l'Australie a accordé ce droit à ses ressortissants. Aujourd'hui, un grand nombre d'États donnent ce droit à leurs citoyens expatriés $^{10}$. L'Iran fait partie des pays où il

6. La question de la migration des Iraniens au Canada est étudiée dans cet article à partir des bases de données encyclopédiques. Pour ce qui est de la question des relations diplomatiques irano-canadiennes, je me suis fondée sur des données médiatiques. En ce qui concerne le concept des élections à distance, les travaux de Jean-Michel Lafleur m'ont été d'une grande utilité. La question des élections, comme vecteur de mobilisation de la diaspora iranienne, a rarement été abordée. Cela a limité ma recherche aux médias, blogs et réseaux sociaux. J'ai également mené des entretiens téléphoniques avec certains membres de la diaspora iranienne à Toronto. 7. www.canadainternational.gc.ca/iran/canada-iran/direct diplomacy-diplomatie_directe.aspx?lang=fra 8. Bahador Zabihiyan, "Ottawa a créé un vaste réseau d'opposants iraniens", in Le Devoir, 16 juin 2014. 9. "Discours du ministre Baird au Global Dialogue on the Future of Iran", sur le Site officiel du gouvernement du Canada, 10 mai 2013. 10. Jean-Michel Lafleur, “Le droit de vote à distance : émergence d'un objet de recherche et apports au champ de la sociologie électorale", in Colloques et congrès scientifiques, octobre 2015. 
a été accordé relativement tôt ${ }^{11}$, en $1980^{12}$. Depuis, les citoyens expatriés iraniens peuvent voter à distance aux scrutins présidentiels, référendaires et infranationaux de leur pays d'origine. Le fait que la diaspora se soit vue accorder ce droit dès la deuxième année de la révolution iranienne semble indiquer que le régime iranien a eu vite conscience de l'importance de la participation de ses citoyens expatriés aux événements politiques du pays.

Bien que l'Iran compte parmi les pays qui attribuent le droit de vote à distance à leur diaspora, il n'a pourtant toujours pas mis en place d'initiatives telles que le vote électronique ou le vote postal. Cela signifie que la présence personnelle des électeurs aux scrutins lors d'une élection est nécessaire. Selon l'article 53 de la loi concernant les élections présidentielles iraniennes, les ambassades, les consulats ou les représentants diplomatiques du régime de la République islamique ont la charge du déroulement des élections à l'étranger. Ainsi, l'ambassade d'Iran à Ottawa organisait des scrutins pour les Iraniens sur place désireux de participer aux élections iraniennes. Or, suite à sa fermeture après la rupture des relations diplomatiques irano-canadiennes, aucune structure officielle ne pouvait plus assurer une telle organisation. Les Iraniens désireux de voter devaient renoncer à leur droit lors de l'élection présidentielle de 2013.

Les gouvernements des deux pays se sont renvoyé la responsabilité de cette participation rendue impossible. Dans une déclaration, le ministre canadien des Affaires étrangères accuse le gouvernement iranien de "n'avoir pris aucune mesure pour que les Iraniens au Canada puissent participer aux élections ${ }^{13}$ ". Le gouvernement iranien a, quant à lui, répondu que les autorités canadiennes n'avaient pas souhaité "coopérer" avec l'Iran dans la mise en place d'un scrutin au Canada. La querelle des deux gouvernements sur la responsabilité de l'organisation de ce scrutin a au final privé de vote les Iraniens du Canada. En comparaison, alors que l'Iran n'a pas maintenu de relations diplomatiques avec les États-Unis et qu'il n'y dispose pas d'ambassade, des scrutins y ont néanmoins été organisés pour permettre aux Iraniens sur place de voter. Malgré ces relations dégradées avec l'Iran, les États-Unis ont ratifié l'organisation des élections iraniennes sur leur territoire ${ }^{14}$.

L'absence de bureau de vote au Canada a conduit plusieurs Iraniens du Canada désireux de voter à lancer des initiatives afin de montrer leur Selon l'article 53 de la loi concernant les élections présidentielles iraniennes, les ambassades, les consulats ou les représentants diplomatiques du régime de la République islamique ont la charge du déroulement des élections à l'étranger. volonté de participer au processus électoral. Tel le fait d'encourager les Iraniens qui souhaitent s'abstenir pour sanctionner le régime à "donner" leur vote. Ce statut diffusé par une Iranienne sur sa page Facebook ainsi que sur une autre page intitulée "Les élections et nous" en témoigne : "Les amis, je suis une Iranienne résidente au Canada qui ne peut pas participer aux élections. En raison de la rupture des relations diplomatiques entre les deux pays, il n'y a actuellement aucun scrutin organisé au Canada pour les élections présidentielles iraniennes. S'il y a quelqu'un parmi les amis iraniens en Iran ou dans un autre pays [où les Iraniens sont en mesure de voter] qui boycotte les élections, mais qui respecte le point de vue opposé, je voudrais lui demander de voter à ma place ${ }^{15}$." De pareilles demandes ont été nombreuses, suscitant beaucoup de débats. Elles ont souvent débouché sur des réponses positives et les "boycotteurs" ayant ainsi voté ont posté une photographie de leur bulletin en guise de preuve.

La mise en place d'un système de covoiturage, afin d'aller voter à la Section des intérêts iraniens de Washington ou de New York, a constitué un autre moyen de participer aux élections, mais avec un

11. Considérons qu'au Moyen-Orient ou même dans certains pays européens, soit ce droit n'est toujours pas donné, soit il est donné majoritairement après l'année 2000. 12. "External voting : a world survey of 2014 countries and territories", in International IDEA, 2014. 13. www.international.gc.ca/media/aff/speeches-discours/2013/o5/10a.aspx?lang=fra. 14. Voir Jean-Michel Lafleur, Transnational Politics and the State, Londres, Routledge, 2012. Dans cet ouvrage, l'auteur aborde la question de la politique des pays d'immigration face au droit de vote à distance des immigrés présents sur leur territoire. II semble que le Canada, bien qu'il soit un pays multiethnique, n'est pas très à l'aise avec ce vote : "Le Canada craint que les questions politiques stimulent des tensions ethniques au sein de certaines diasporas, et que ça nuise au bon vivre-ensemble de la société canadienne." 15. www.facebook.com/events/600012370024118. 
succès moindre en raison du coût et du temps du voyage, ainsi que des complications administratives liées au statut des Iraniens résidant au Canada.

\section{Une élection symbolique}

L’organisation d'élections symboliques" a été mise en place par un groupe de jeunes iraniens habitant notamment Toronto ${ }^{16}$, grâce aux réseaux sociaux, avec, par exemple, la page Facebook "Les élections et nous", qui a offert à l'événement une large publicité. Mais les militants ont également été actifs sur le terrain, placardant dans la ville des affiches portant les messages suivants: "L'indifférence ne changera pas le régime politique actuel" et "Avoir un vote est notre droit, exigeons ce droit civil et politique. Les gouvernements iranien et canadien

L'organisation d'“élections

symboliques" a été mise

en place par un groupe

de jeunes iraniens habitant

notamment Toronto, grâce

aux réseaux sociaux, avec,

par exemple, la page Facebook

"Les élections et nous",

qui a offert à l'événement une large publicité doivent reconnaitre notre droit en tant que peuple iranien à déterminer notre propre avenir".

Ces élections symboliques eurent lieu du 10 au 13 juin à Toronto. Quatre bureaux de vote symboliques ont été installés dans les endroits fréquentés par les Iraniens de la ville. Les participants à ces "élections symboliques" recevaient un bulletin de vote qui contenait le nom des huit candidats à la présidence iranienne, ainsi que les options: "Je ne voterais pas même si j'avais accès aux scrutins" et "Autre choix".

Arash Abadpoor, un des organisateurs des élections symboliques de Toronto, explique les objectifs de la campagne: "Nous sommes un groupe d'Iraniens de Toronto n'ayant pas nécessairement le même point de vue sur toutes les questions liées à l'Iran, mais nous avons évidemment beaucoup de points de vue communs. Un des points sur lequel nous sommes tous d'accord, c'est que le changement ne survient que lorsque le peuple l'exige et participe aux processus qui le permettent. Nous croyons que le boycott d'une élection est (...) peut-être une approche romantique et idéaliste ; néanmoins, pour pouvoir faire partie de la solution, les gens doivent utiliser leur droit de vote ${ }^{17}$."

Arash Abadpoor était pourtant l'un des rares invités de la conférence pour "le dialogue global pour l'avenir de l'Iran" organisée par le ministère des Affaires étrangères canadien. Qu'il ait jugé plus utile de rejoindre les initiateurs des élections symboliques à Toronto, en contradiction avec la volonté de délégitimation du processus électoral en Iran de la part du gouvernement canadien, illustre bien l'échec de ce dernier à rallier l'ensemble des militants des droits civils iraniens présents au Canada. Lors de son interview à Radio Farda, Abadpoor critique cette politique de suspension des relations diplomatiques entre l'Iran et le Canada : "Nous [le groupe organisateur des élections symboliques] tenions à nous plaindre du fait qu'aucun moyen de voter ne soit offert aux Iraniens du Canada. La fermeture de l'ambassade d'Iran au Canada résulte du contentieux politique entre les deux pays, mais répond aussi aux voeux de certains Iraniens présents au Canada. Cet événement, à notre avis, a causé des difficultés pour les affaires quotidiennes des Iraniens, telles que le renouvellement d'un passeport ou l'obtention d'un acte de naissance. Et, à plus grande échelle, [cette rupture] a retiré au gouvernement canadien et aux Iraniens du Canada la possibilité d'interagir avec le gouvernement iranien. Si l'ambassade iranienne était ouverte, on aurait pu faire nos demandes en présence des représentants officiels du gouvernement d'Iran ${ }^{18}$."

\section{Le réveil d'une conscience nationale}

Les résultats de ces élections, diffusés sur les réseaux sociaux, sont éclairants : sur 701 suffrages exprimés, 387 correspondaient à l'option : "Je ne voterais pas même si j'avais accès aux scrutins", alors que 114 participants avaient voté pour Hassan Rohani, 
le président élu. Cela montre que même si la majorité ne comptait pas participer au scrutin présidentiel, elle a tenu à manifester par ce vote symbolique son droit à voter et à s'abstenir. Certaines personnes n'auraient probablement pas voté si des élections régulières avaient pu être organisées. Les participants à ces élections symboliques ont voulu protester contre ce qui revenait à faire d'eux des "invisibles". Il s'agissait également d'une manière de protester contre cette rupture décidée de manière impulsive par le gouvernement canadien, et qui les touche dans leur vie quotidienne. Mahmoud Azimaee, un autre organisateur de ces élections symboliques, qualifie cette élection d'“inoubliable expérience". Il précise que le résultat n’a pas déçu les organisateurs, car le but était de pratiquer la démocratie. Mais il proteste contre le fait que les Iraniens du Canada n'aient pu choisir de participer ou non aux élections par eux-mêmes : "Avec la rupture des relations diplomatique, on ne bénéficie plus des services consulaires. En ce qui concerne les élections, on n'a ni le droit de boycotter, ni le droit de voter : on est condamné à ne pas voter ${ }^{19}$." Certains des organisateurs de ces initiatives sont des Iraniens émigrés de longue date ou des opposants au régime interdits de retour en Iran. En temps normal, ces élections auraient dû, pour bien d'entre eux, les laisser au mieux indifférents. L'impossibilité d'y participer, imposée par les mesures politiques prises par leur pays d'accueil, le Canada, a pour ainsi dire réveillé chez eux une conscience nationale, une appartenance à un peuple iranien qui inclut à la fois les Iraniens d'Iran et les Iraniens de la diaspora.

\section{Les soutiens iraniens à la position canadienne}

L'importante diaspora iranienne au Canada est cependant loin d'être unanime quant à la rupture des relations diplomatiques avec l'Iran. Certains en ont même été enchantés et ont organisé leurs propres manifestations de soutien au gouvernement canadien. Un groupe d'activistes iraniens, Iranian Liberal Students and Graduates, a organisé à Toronto une manifestation dont Salman Sima, l'un des organisateurs, explique l'objectif : "Notre

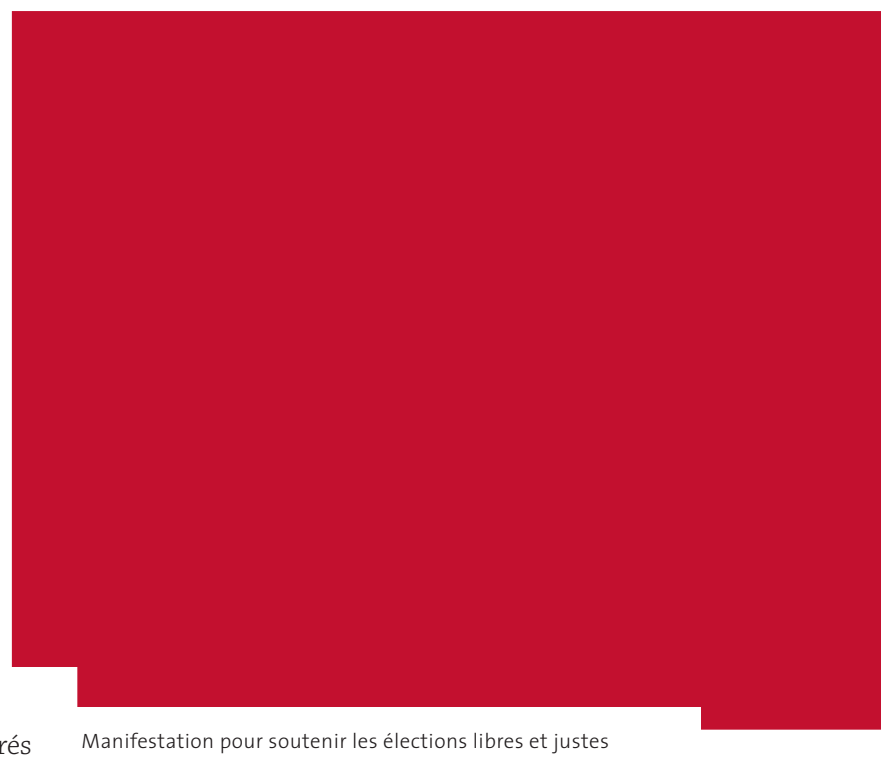
en Iran. (C) LeILA KoochaKZADEH.

but était de soutenir des élections libres et justes. Les élections organisées en Iran ne sont en aucune manière compatibles avec les normes pour des élections libres et équitables ${ }^{20}$."

Le 8 juin 2013, une quarantaine d'Iraniens se sont rassemblés dans un des quartiers les plus fréquentés par le public canadien, "afin d'attirer l'attention des Canadiens sur la question des élections non démocratiques en Iran". Les pancartes portaient des slogans en anglais uniquement (et non en persan), tel que : "Isolate Islamic Republic of Iran" ou "Help Iranian People Topple the Regime [sic] $]^{21 "}$.

Cette manifestation aurait vraisemblablement été décidée à la suite d'une réunion du Conseil natio- 
nal d'Iran (CNI), le 3 juin 201322. Reza Pahlavi, fils du roi déchu Mohammad Reza Shah Pahlavi, s'était rendu à Toronto en tant que porte-parole du CNI pour prononcer un discours au sujet de l'élection présidentielle en Iran, dans lequel il a vivement défendu la position du gouvernement canadien envers l'Iran : “Aujourd'hui, le Canada fait partie des principaux pays ayant rompu toutes ses relations diplomatiques avec le régime dictatorial de la République islamique, afin de soutenir les droits de l'Homme. Il revient à nous, le peuple iranien, de remercier dirigeants et diplomates canadiens, qui ont entendu la voix du peuple iranien, pour cette action humanitaire ${ }^{23}$."

Au cours de cette réunion, le CNI a décidé de faire de la semaine du 7 au 14 juin 2013 "la semaine des élections libres". La ressemblance entre les slogans et les mots d'ordre décidés au cours de cette réunion et lors de la manifestation pour les "élections libres" laissent penser que cette dernière a également été décidée

Le 15 juin 2013, des Iraniens se sont rassemblés devant

le parlement, à Ottawa, pour célébrer le résultat des élections, alors que beaucoup d'Iraniens d'Iran festoyaient

dans les rues de Téhéran et des autres grandes villes iraniennes. par le Conseil. Dans les discours des Iraniens du Canada favorables aux élections, nous retrouvons régulièrement une frustration tant à l'égard de la rupture des relations canadiennes avec l'Iran qu'envers les opposants les plus extrémistes au régime iranien, qui encouragent le gouvernement canadien à la fermeté. Pour ces derniers, la rupture des relations diplomatiques pourrait être un prélude à l'isolement total de l'Iran qu'ils appellent de leurs vœux. Cette solution pourrait être la seule à même de renverser le régime islamique et de "sauver l'Iran et les Iraniens". À l'inverse, les Iraniens "pro-élections" ne sont que bien rarement des partisans du régime en place en Iran. Le clivage est ailleurs et se situe dans la conviction que c'est au peuple iranien de choisir son destin et de provo- quer les changements politiques nécessaires, sans intervention des autres pays.

\section{Désaccords autour de l'élection d'Hassan Rohani}

Au lendemain de l'élection d'Hassan Rohani, une grande partie du peuple iranien a manifesté sa joie, y compris au Canada. Le 15 juin 2013, des Iraniens se sont rassemblés devant le parlement, à Ottawa, pour célébrer le résultat des élections, alors que beaucoup d'Iraniens d'Iran festoyaient dans les rues de Téhéran et des autres grandes villes iraniennes. Une manière de communier avec le peuple iranien, après le vote même, dans la joie devant les résultats ${ }^{24}$.

Le gouvernement canadien a continué à mener sa politique de fermeté envers l'Iran. Suite à l'annonce des résultats de l'élection présidentielle iranienne, et malgré la réaction positive des autres chancelleries occidentales, John Baird les a qualifiés d'imposture". Fustigeant les contraintes internes qui pèsent en Iran sur l'organisation d'élections réellement libres, il a traité notamment les huit candidats à l'élection présidentielle, dont le président élu, Hassan Rohani, de "marionnettes entre les mains des ayatollahs" : "Le Canada salue le courage des Iraniens qui ont exprimé leur désir de liberté face à une répression impitoyable. Étant donné la manipulation de la volonté collective et du processus démocratique par le régime actuel, les résultats du scrutin du 14 juin ne veulent rien dire dans les faits ${ }^{25}$." Ces déclarations ont provoqué la protestation d'un certain nombre de membres de la diaspora iranienne au Canada. Aida Ahadiany, une IranoCanadienne, a demandé sur son blog, à ses lecteurs, de renvoyer au ministre des Affaires étrangères du Canada la lettre de protestation quelle avait elle-même écrite et envoyée : "Vous avez qualifié la participation des Iraniens à leur élection d"impos- 
ture’. Je suis moi-même irano-canadienne et demeure en contact avec mes amis et ma famille restés en Iran. Des gens qui ont œuvré des heures durant pour que la récente campagne en faveur du vote ait lieu. De ceux qui souhaitaient combattre l'extrémisme et la violence et qui, contre toute attente, ont gagné. Quoique, de manière inattendue, la Maison Blanche, le président Obama lui-même, et bien des gouvernements européens aient salué la volonté démocratique du peuple iranien exprimée dans ce pas important vers le changement. Face à tant de soutien, il est décevant pour les citoyens irano-canadiens comme nous, qui participons également aux processus électoraux canadiens, de voir notre contribution démocratique considérée comme une 'imposture'. M. Baird, je vous écris ce message afin que vous puissiez comprendre et reconnaître l'espoir du peuple iranien dans le changement, et le soutenir dans ce cheminement difficile ${ }^{26}$." Cette initiative a été massivement soutenue par les Iraniens qui ont renvoyé en grand nombre cette lettre à $\mathrm{M}$. Baird ainsi qu'à Paul Dewar, député néo-démocrate ayant estimé que les propos du ministre des Affaires étrangères canadien constituaient "une gifle au visage du peuple iranien ${ }^{27 "}$.

Devant le tollé provoqué, le ministre canadien des Affaires étrangères a dû faire marche arrière le 17 juin $^{28}$, date à laquelle il publia une lettre d'excuse dans le journal Globe and Mail : "Salam, Le Canada a entendu la voie de tous ceux, à l'intérieur de l'Iran, qui ont voté pour l'espoir et un meilleur avenir pendant l'élection présidentielle iranienne. Il revient au seul peuple iranien de donner une signification à cette élection. (...) Tout comme nous respectons les voix des militants courageux qui croient que le régime est incapable de changer, nous honorons et respectons les voix de ceux qui croient que cette élection peut être une étape vers le changement démocratique fondamental en Iran. Je les félicite ${ }^{29}$." Un des enjeux pour certains membres de la diaspora iranienne est de s'affirmer malgré tout comme Iraniens, au-delà de l'éloignement géographique ou de la citoyenneté canadienne de certains. C'est là une forme de fierté nationale de la part de cette diaspora que l'on peut constater à la fois dans l'indignation que suscitent les leçons de morale démocratique du gouvernement canadien quant au processus électoral iranien, et dans la revendication d'une expression démocratique partiellement retrouvée à travers l'élection d'Hassan Rohani.

\section{Conclusion}

En mettant en lumière la question des relations diplomatiques irano-canadiennes ainsi que le concept de l'octroi du droit de vote à distance par l'État iranien à ses citoyens expatriés, il s'agissait d'étudier la réaction de la diaspora iranienne face à la suspension des relations de deux pays, en se focalisant sur la question de l'élection présidentielle iranienne de l'année 2013. Un grand nombre d'Iraniens résidant au Canada, voire à l'étranger, habitent à Toronto. Contrairement aux Iraniens de Los Angeles qui forment une communauté avec une origine sociale "bien délimitée $e^{30 ", ~ l e s ~ I r a n i e n s ~ d e ~}$ Toronto représentent différentes parties du spectre sociopolitique de leur pays d'origine. L'affaire qui a eu lieu à Toronto en 2013 autour de l'élection présidentielle iranienne illustre tout l'intérêt que continuent de porter les Iraniens émigrés à la situation et à l'évolution politiques de leur pays d'origine. Plus encore, elle manifeste de leur part une volonté de participation à sa vie politique, même hors de ses frontières, quitte à braver des situations difficiles. Cette diaspora apparaît néanmoins profondément divisée, entre les opposants radicaux au régime de la République islamique partisans d'un renversement de celui-ci par la force et les partisans d'une solution réformiste qui passe par la participation aux élections organisées par le régime. Malgré ces divisions, la capacité d'influer sur la politique du gouvernement canadien vis-à-vis de l'Iran, dans un sens comme dans l'autre, montre la force de la diaspora iranienne présente au Canada. 